\title{
COMPARAÇÃO DE MÉTODOS DE CLASSIFICAÇÃO DE IMAGENS NA IDENTIFICAÇÃO DE ÁREAS CULTIVADAS COM CITROS ${ }^{1}$ \\ ANA PAULA BARBOSA ${ }^{2}$, SÉRGIO CAMPOS ${ }^{3}$, CÉLIA REGINA LOPES ZIMBACK ${ }^{4}$ \& ZACARIAS BARROS XAVIER ${ }^{5}$
}

RESUMO: A classificação de imagens é o processo de extração de informações para reconhecer padrões e objetos homogêneos em produtos de sensoriamento remoto para mapear temas de interesse em áreas na superfície terrestre. Esse trabalho teve por objetivo comparar a eficiência de métodos de classificação de imagens orbitais em áreas cultivadas com citros. A área de estudo utilizada foi a Fazenda Água Branco município de Bariri/ SP. O SIG - Idrisi 15.0 foi utilizado no processamento das imagens do satélite LANDSAT-5 TM, órbita/ponto 221/75, passagens de 26/05/2007. No trabalho realizado constatou-se que os resultados da avaliação de acurácia da classificação foram satisfatórios, sendo que a classificação do algoritmo CLUSTER apresentou qualidade muito boa $(0,6485)$ e o algoritmo MAXVER apresentou classificação excelente, com Kappa de 0,8818. Os métodos utilizados para a discriminação das áreas cultivadas com citros mostraram eficiências distintas na classificação das imagens. De modo geral, as classificações apresentaram desempenhos satisfatórios e em termos de comparação de áreas, o classificador CLUSTER foi o que melhor se aproximou da área real.

Palavras-chave: LANDSAT, classificadores de imagens, SIG.

\footnotetext{
${ }^{1}$ Parte da dissertação de Mestrado do $1^{\circ}$ autor intitulada: "Comparação de métodos de classificação de imagens, visando o gerenciamento de áreas citrícolas”. Defesa: 14/08/2009

${ }^{2}$ Doutoranda em Agronomia - Energia na Agricultura, FCA/UNESP, Botucatu/SP, Brasil, Engenheira Agrônoma. e-mail: anap_barbosa@yahoo.com.br

${ }^{3}$ Docente do Departamento de Engenharia Rural, FCA/UNESP, Botucatu/SP, Brasil, e-mail: seca@fca.unesp.br

${ }^{4}$ Docente do Departamento de Recursos Naturais - Ciência do Solo, FCA/UNESP, Botucatu/SP, Brasil. e-mail: czimback@gmail.com

${ }^{5}$ Docente do Departamento de Engenharia Rural, FCA/UNESP, Botucatu/SP, Brasil, e-mail: labaero@fca.unesp.br
} 


\section{COMPARING METHODS OF IMAGE CLASSIFICATION TO IDENTIFY CULTIVATED CIT- RUS ÁREAS}

SUMMARY: Image classification is the process of extraction of information to recognize patterns and homogeneous objects in remote sensing products to map themes of interest in areas of the surface. This study aimed to compare the efficiency of classification methods of orbital images in areas cultivated with citrus. The study area is located at Água Branca Farm, Bariri City/SP. The GIS - Idrisi 15.0 was used in the image processing of TM LANDSAT-5 satellite images, path/row 221/75, 05/26/2007. The evaluation results of the classification accuracy were satisfactory and the classification of the CLUSTER algorithm had very good quality (0.6485) and the MAXVER algorithm had excellent rating, with kappa of 0.8818 . The methods used for the discrimination of areas cultivated with citrus showed different efficiencies in the classification of images. The classifications had satisfactory performance and the CLUSTER classifier was the one that is closer to the real area.

Keywords: LANDSAT, Images classifiers, GIS.

\section{INTRODUÇÃO}

A citricultura no Estado de São Paulo é de grande importância econômica e social, pois apresenta participação significativa na geração de empregos, na ocupação da terra, formação de renda, ativação dos elos da cadeia produtiva, captação de divisas e desenvolvimento regional de outros setores da economia (prestação de serviços, transportes, comércio, etc.).

O Estado de São Paulo produziu em 2007, 14.904 .621 toneladas de laranja, um acréscimo de 3,7\% comparado ao ano de 2006 (IBGE, 2007). Porém a incidência de doenças como o cancro cítrico, pinta preta, morte súbita (MSC), clorose variegada dos citros (CVC) e o greening, preocuparam bastante os técnicos e produtores (IBGE, 2007).

Com a importância econômica da citricultura para o país, o estudo de pomares de citros a partir de sensores de resolução espacial equivalente ao TM/LANDSAT exige o entendimento de diversos fatores. Isto porque, ocorrem variações na resposta espectral destes alvos em função de alterações no substrato, altura e cobertura do terreno pelas árvores e características geométricas da disposição das plantas e das condições de observação do sensor (COVRE,1989).

Freitas Filho e Medeiros (1993) constataram que as vantagens de se utilizarem dados de sensoriamento remoto nos levantamentos do uso das terras visam atingir áreas de difícil acesso e fazer o imagea- 
mento de grande altitude, possibilitando uma visão sinóptica da superfície terrestre, com repetitividade, viabilizando o monitoramento de grandes áreas.

Moreira (2001) relatou que ao incidir sobre um alvo, a radiação proveniente do sol, interage com o seu material constituinte. A irradiância que incide numa superfície pode ser parcialmente refletida, absorvida ou transmitida. Os objetos da superfície terrestre como a vegetação, a água e o solo refletem, absorvem e transmitem radiação eletromagnética em proporções que variam com o comprimento de onda, de acordo com as suas características bio-físico-químicas (FLORENZANO, 2002).

O comportamento espectral refere-se à curva de reflectância em função do comprimento de onda nas regiões do espectro eletromagnético. O comportamento espectral da vegetação é caracterizado por três regiões distintas: visível, infravermelho próximo e infravermelho médio (MOREIRA, 2001).

Sensores multiespectrais registram simultaneamente várias faixas largas do espectro, gerando imagens da mesma cena. Estas imagens multiespectrais ampliam a possibilidade de se extrair informações de um objeto ou de uma área na superfície, pois permitem analisar o comportamento espectral de um alvo nas distintas bandas do espectro. A maioria dos sensores multiespectrais utiliza de três a seis bandas espectrais, abrangendo a região do visível ao infravermelho médio (NOGUCHI, 2004).

O processamento digital de imagens, de uma maneira geral, tem como função primordial o desenvolvimento e a utilização de técnicas para identificar, extrair, condensar e realçar informações de interesse para determinados fins, a partir de uma enorme quantidade de dados que usualmente compõem a imagem (GONÇALVES, 1997).

A classificação de imagens em sensoriamento remoto é a identificação de feições na imagem, isto é, a tradução dos diversos padrões de energia eletromagnética refletida em classes de cobertura terrestre (LILLESAND, KIEFER, 1994).

Considera-se a classificação não supervisionada um procedimento em que não há interação entre o sistema e o analista. Durante a fase de treinamento, certos algoritmos necessitam de alguns parâmetros que devem ser fornecidos ao sistema (MOREIRA, 2001).

A classificação supervisionada é o procedimento utilizado com maior freqüência para análise quantitativa de dados de imagens de sensoriamento remoto. Neste processo são utilizados algoritmos para nomear os pixels em uma imagem de forma a representar tipos específicos de cobertura terrestre, ou classes que são conhecidas a priori (LILLESAND, KIEFER, 1994).

A fim de aprimorar as técnicas de classificação, são empregadas regras para discriminar alvos em imagens de média resolução espectral, visando obter, de forma acurada, informações confiáveis dos recursos naturais. A proposta deste trabalho foi comparar diferentes métodos de classificação de imagens de satélite, a fim de identificar o método que melhor se aplica à classificação de áreas citrícolas. 


\section{MATERIAL E MÉTODOS}

\subsection{Material}

A área de estudo foi à região da Fazenda produtora de citros Água Branca, localizada no município de Bariri, SP, situada entre as latitudes $22^{\circ} 01^{\prime} \mathrm{S}$ a $22^{\circ} 59^{\prime} \mathrm{S}$ e longitudes $48^{\circ} 47^{\prime} \mathrm{WGr}$ a $48^{\circ} 45^{\prime} \mathrm{WGr}$ (Figura 1), totalizando 320,0 ha de área plantada, aproximadamente.

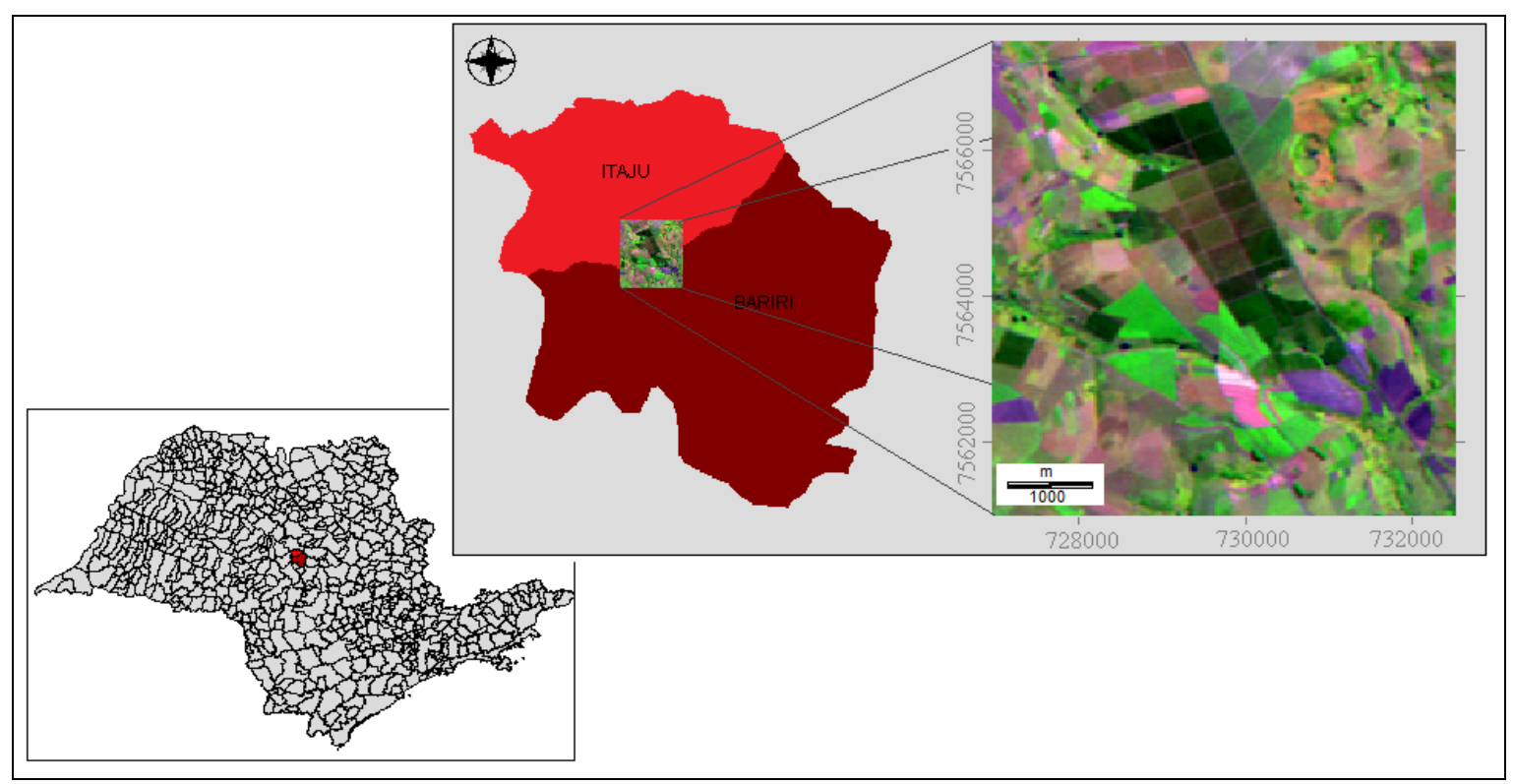

Figura 1 - Localização da Fazenda Água Branca, Bariri - SP

Na propriedade são cultivadas as variedades de citros: Hamlin, Natal, Pera, e Valencia, totalizando 28 talhões. Desse total, cinco talhões da variedade Pera foram erradicados por razões fitossanitárias no ano de 2006.

O solo predominante na região foi classificado como Latossolo Vermelho (IPT, 1981), apresentando o relevo do tipo suavemente ondulado. O clima predominante na região é Cwa, segundo a classificação de Köppen, tropical úmido com inverno seco, temperatura média do mês mais frio inferior a $18^{\circ} \mathrm{C}$ e a do mês mais quente $22^{\circ} \mathrm{C}$; o total das chuvas do mês mais seco não ultrapassa $30 \mathrm{~mm}$ e o índice pluviométrico varia entre 1.100 e $1.700 \mathrm{~mm} \mathrm{ano}^{-1}$.

Foi utilizada imagem do satélite LANDSAT-5 TM, correspondente a órbita 221, ponto 75, em composição falsa cor RGB 543, obtida gratuitamente na página de catálogo de imagens CBERS (INPEDGI, 2008), referente à data de passagem do dia 26/05/2007. 
Para processamento das imagens foi utilizado o Sistema de Informação Geográfica (SIG) Idrisi 15.0 - Andes Edition (EASTMAN, 2006).

\subsection{Metodologia}

As imagens foram analisadas e comparadas quanto às classificações: interpretação VISUAL, não supervisionada CLUSTER e supervisionada MAXVER.

A metodologia para o processamento das imagens foi baseada na descrição de Eastman (2006). Na fase de pré-processamento, as imagens foram preparadas para a realização da classificação. Esta é uma etapa importante, pois visa melhorar a qualidade dos dados, com o emprego de algumas técnicas, como: realce da imagem; redução da dimensionalidade, retificação geométrica e registro.

Para correção dos erros contidos na imagem devido à movimentação do satélite e curvatura da Terra (projeção e sistema de referência) foi executada a retificação geométrica. Neste processo, fez-se o registro utilizando coordenadas geográficas dos pontos de controle, utilizando uma imagem previamente registrada, onde pontos devidamente identificados (georreferenciados) foram associados aos pixels da imagem de interesse.

Para a execução das classificações, as bandas selecionadas foram TM3, TM4 e TM5, pois, correspondem às regiões do espectro eletromagnético que melhor representam alvos agrícolas importantes para o planejamento.

Como técnica de classificação, foi utilizado o método de classificação supervisionada MAXVER, discriminando cinco classes de uso do solo na imagem: citros, solo exposto, cana-de-açúcar, cultura anual e pastagem. Em seguida, foram digitalizados polígonos em torno dessas áreas, atribuindo um identificador para cada tipo de cobertura.

Cada classe de treinamento foi definida com um número mínimo de 30 pixels, já que o número de pixels não deve ser menor do que dez vezes o número de bandas classificadas para cada conjunto de treinamento, onde estes são todas as áreas de treinamento para uma mesma classe de cobertura do solo (Eastman, 2006).

Após definidas as áreas de treinamento, os pixels nelas contidos foram analisados e assinaturas espectrais foram criadas para cada objeto. Finalmente, executou-se o classificador, onde todos os pixels foram atribuídos com igual probabilidade para cada classe de uso.

Também se realizou o processo de classificação não supervisionada, utilizando o algoritmo CLUSTER, que toma como base uma imagem composta, para obtenção de agrupamentos satisfatórios. As bandas para classificação foram selecionadas e posteriormente, foi escolhido o nível de generalização do tipo FINE para obtenção de uma imagem com maior número de agrupamentos. 
Selecionou-se a opção para eliminação de agrupamentos não significativos, obtendo-se o agrupamento de cinco clusters representativos para avaliação.

Depois de identificadas as classes espectrais referentes à classe de uso citros, elas foram agrupadas, desconsiderando-se as demais classes. O agrupamento das classes espectrais obtidas nas classificações foi realizado com auxílio da ferramenta "Create Boolean for current class”, do IDRISI Andes. Essa ferramenta facilita a identificação e associação das classes espectrais resultantes da classificação, tendo como resultado uma imagem binária da área de citros para cada classificação, onde as classes espectrais correspondentes a citros receberam o valor um (1) e as demais o valor zero (0).

Para avaliação dos resultados gerados nas classificações foram efetuados mapas de referência, elaborados a partir da interpretação em tela da cena dimensionalisada.

Para cada data foi gerado um mapa de referência, com os quais foram feitas as tabulações cruzadas entre as imagens classificadas e a referência, obtendo-se o índice Kappa.

Tabela 1 - Qualidade da classificação associada aos valores da estatística Kappa

\begin{tabular}{ll}
\hline Valor de Kappa & Qualidade do mapa temático \\
\hline$<$ zero & Péssima \\
Zero $-0,20$ & Ruim \\
$0,20-0,40$ & Razoável \\
$0,40-0,60$ & Boa \\
$0,60-0,80$ & Muito Boa \\
$0,80-1,0$ & Excelente
\end{tabular}

Fonte: Adaptada de Landis e Koch, 1977, citado por MOREIRA (2001).

Considerando que valores próximos ao número 1 indicam plena coincidência entre a verdade terrestre e o resultado da classificação, enquanto que, um valor próximo a 0 sugere que o resultado é puramente gerado ao acaso. O coeficiente Kappa é o método mais indicado e conhecido para aferição das classificações temáticas (MOREIRA, 2001).

\section{RESULTADOS E DISCUSSÃO}

A avaliação do desempenho das classificações de imagens requer a elaboração de um mapa de referência, que foi obtido a partir da interpretação em tela da área de estudo, da imagem de satélite do dia 26 de maio de 2007. 
Seis (6) classes de uso foram determinadas: citros, solo exposto, cana-de-açúcar, cultura anual, pastagem e vegetação nativa. As demais classes de uso não foram contempladas devido às pequenas áreas presentes nas imagens analisadas (Figura 2).

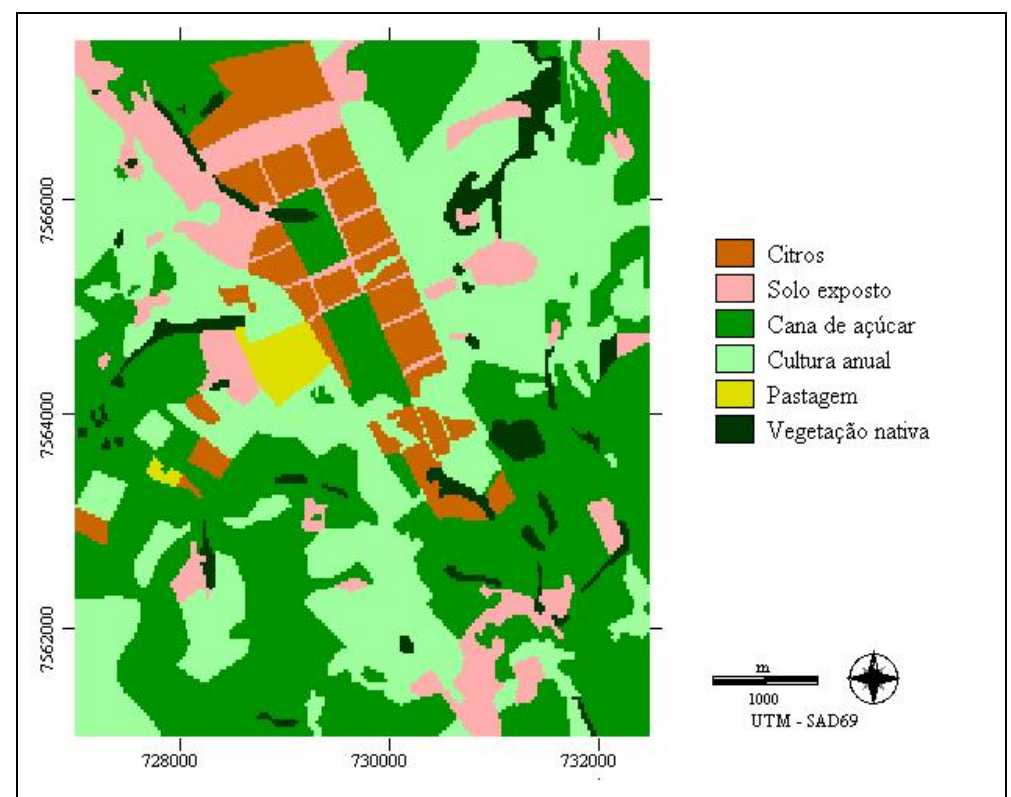

Figura 2 - Interpretação visual obtida da passagem do satélite LANDSAT-5 TM em 26/05/2007.

Os demais métodos de classificação foram avaliados com base no resultado obtido da interpretação visual, representando a verdade terrestre.

O método não supervisionado CLUSTER tem como produto a identificação de classes espectrais distintas na imagem, as quais podem ser identificadas como tipos de cobertura por meio da associação com dados de referência terrestre.

Observando o mapa obtido por este classificador, quando comparado ao mapa de referência (Figura 2), foi possível detectar nitidamente que os pomares citrícolas sofreram influência de outras classes na sua resposta espectral, pois parte do pomar não foi classificado como tal, embora as áreas agrupadas como citros tenham sido detectadas com definição, discriminando-as das demais classes (Figura 3). 


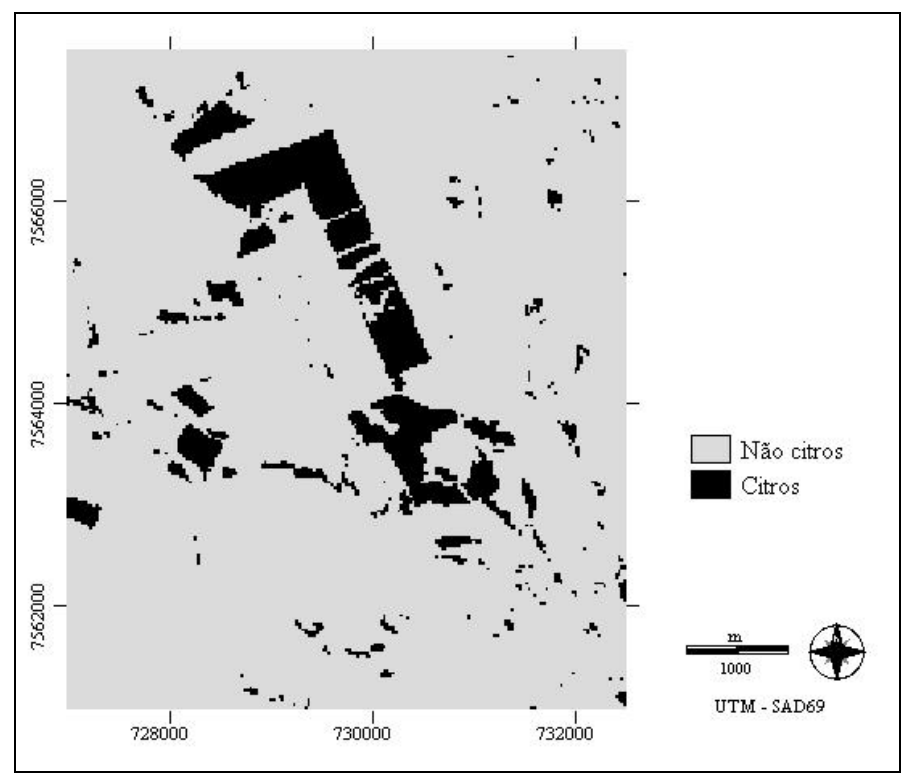

Figura 3 - Classificação CLUSTER obtida da passagem do satélite LANDSAT-5 TM em 26/05/2007.

Alguns erros de designação do classificador CLUSTER foram observados nas áreas de transição entre culturas anuais, solo exposto e o citros. Embora a classe citros tenha sido bem definida pelo classificador, os talhões erradicados sofreram forte influência da reflectância da classe solo exposto.

Sanches et al. (2008), ao discriminar variedades de citros a partir de imagens CCD/CBERS-2, verificaram que o classificador não supervisionado ISOSEG apresentou resultado satisfatório quando aplicado em época seca.

O classificador MAXVER está implementado nos principais SIG's de processamento digital de imagens digitais, por ser considerado um dos mais eficientes classificadores e é, consequentemente, um dos mais utilizados.

Pelo fato deste classificador utilizar áreas de treinamento definidas pelo analista para agrupar diferentes classes, pode-se verificar que a classe citros foi apropriadamente agrupada, quando comparada com a classificação visual (Figura 4). 


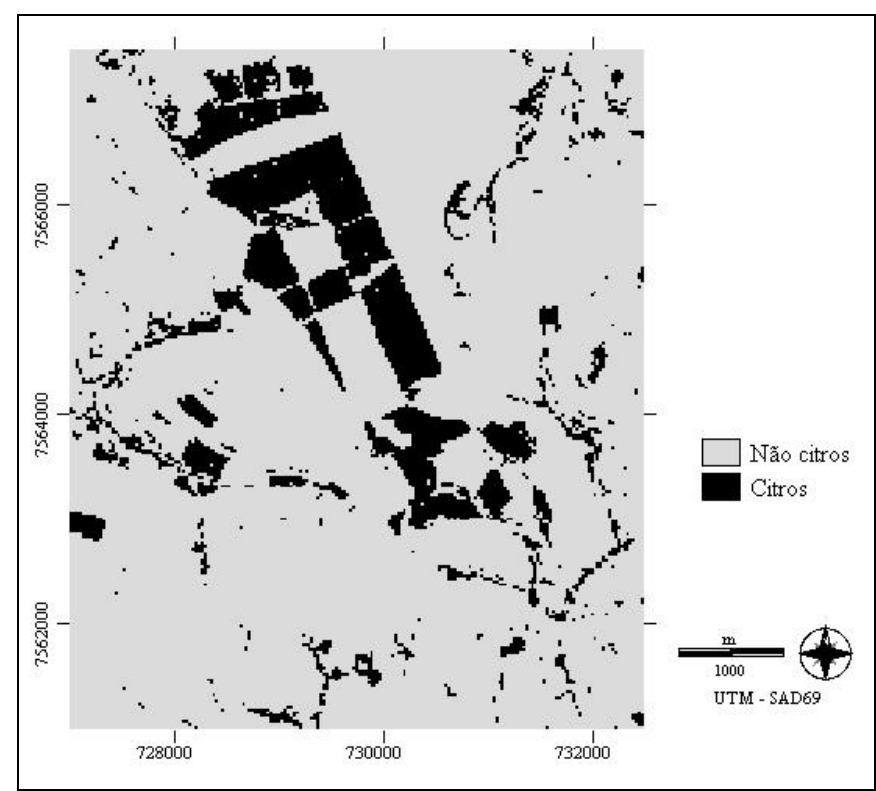

Figura 4 - Classificação MAXVER obtida da passagem do satélite LANDSAT-5 TM em 26/05/2007.

Esse classificador apresentou desenvolvimento satisfatório, inserindo nas classes determinadas os pixels a elas correspondentes. No entanto, observando o mapa de referência, verificou-se que houve confusão do classificador ao agrupar diferentes áreas que apresentavam comportamento espectral semelhante ao desta classe, como o caso da vegetação nativa.

Algumas classes foram suprimidas, pois não alcançaram a quantidade mínima de pixels por área de treinamento, que este caso, é de 30 pixels.

Ao comparar os métodos de classificação, constatou-se que os resultados na avaliação de acurácia da classificação foram satisfatórios, nos quais, a classificação do algoritmo CLUSTER teve qualidade muito boa $(0,6485)$ e classificou como áreas citrícolas 351,54ha (Tabela 2).

O classificador MAXVER apresentou qualidade de classificação excelente, com Kappa de 0,8818, classificando 523,90ha como áreas ocupadas por citros, assim como, Disperati et al. (2003), em estudo da dinâmica florestal de um município obtiveram desempenho geral de classificação excelente (entre 0,9956 e 0,9996).

Celinski e Zimback (2010) também encontraram excelente qualidade de classificação $(0,8099)$, quando utilizaram o MAXVER em imagens de satélite CBERS-2, para as classes temáticas no período de inverno (corpos d’água, solo exposto, eucalipto, pinus, araucária, mata natural, trigo, aveia, pasto e área urbana). 
Tabela 2 - Comparação das classificações CLUSTER e MAXVER, pelo Índice Kappa.

\begin{tabular}{cccc}
\hline Método & Kappa & $\begin{array}{c}\text { Qualidade de classi- } \\
\text { ficação }\end{array}$ & Área (ha) \\
\hline Visual & - & - & 319,62 \\
Cluster & 0,6485 & Muito boa & 351,54 \\
Maxver & 0,8818 & Excelente & 523,90 \\
\hline
\end{tabular}

Embora as classificações não supervisionadas CLUSTER e supervisionada MAXVER tenham apresentado qualidade de classificação variando de muito boa a excelente, foi possível verificar que houve uma discrepância na quantificação das classes.

Quando comparado à classificação visual, o classificador CLUSTER identificou inadequadamente na classe citros 31,92 ha, correspondendo a uma área de 9,98\% superior a área real desta classe. Já no caso do classificador MAXVER, esse valor foi maior em 63,91\%, representados em 204,28ha de área erroneamente classificadas.

Ortiz e Freitas (2005), ao estudar os impactos ambientais de atividades de extração mineral, verificaram que, devido à pequena dimensão das áreas de vegetação natural, classificadores supervisionados superestimaram estas áreas.

Já Góes et al. (2006), utilizando o classificador não supervisionado ISOSEG, verificaram que este subestimou classes de vegetação.

\section{CONCLUSÕES}

Os métodos utilizados para a discriminação de áreas cultivadas com citros mostraram eficiências distintas na classificação das imagens.

O método de classificação não supervisionado CLUSTER mostrou vantagem sobre o outro método, pois classificou as áreas de citros com qualidade e rapidez, não necessitando de intervenção do analista.

O desempenho do método de classificação supervisionada MAXVER foi melhor, embora tenha apresentado diferença significativa entre a área classificada e a verdade terrestre. Além disso, exigiu um elevado tempo na determinação das amostras de treinamento.

Ao comparar os métodos verifica-se que, em termos de comparação de áreas, o classificador CLUSTER foi o que melhor se aproximou da área real. 


\section{AGRADECIMENTOS}

Os autores agradecem à Empresa Citrovita por disponibilizar a área de estudo; ao Conselho Nacional de Desenvolvimento Científico e Tecnológico (CNPq), pelo apoio financeiro e ao Grupo de Estudos e Pesquisas Agrárias Georreferenciadas - GEPAG, pela multiplicação do saber.

\section{REFERÊNCIAS}

CELINSKI. T. M., ZIMBACK, C. R. L. Discriminação de classes de cobertura vegetal utilizando técnicas de classificação digital de imagens de sensoriamento remoto. Revista Energia na Agricultura. Botucatu, v. 25, n.1, p. 152-170, 2010.

COVRE, M. Influencia de parâmetros culturais de citros sobre os dados TM/Landsat. 1989. 241 f.. Dissertação (Mestrado em Sensoriamento Remoto). Instituto Nacional de Pesquisas Espaciais, São José dos Campos, 1989.

DISPERATI, A. A., OLIVEIRA FILHO, P. C., BOBROWSKI, R., DLUGOSZ, F. L. Análise temporal da cobertura florestal do município de Irati-PR, utilizando imagens satelitárias Landsat. Revista Ciências Exatas e Naturais, v. 5, n. 1, p. 115-123, 2003.

EASTMAN, J. R. Idrisi 15: The Andes Edition.Worcester, MA: Clark University, 2006.

FLORENZANO, T. G. Imagens de satélite para estudos ambientais. São Paulo: Oficina de Textos, 2002. $97 \mathrm{p}$.

FREITAS FILHO, M. R.; MEDEIROS, J. S. Análise multitemporal da cobertura vegetal em parte da Chapada do Araripe- CE, utilizando técnicas de sensoriamento remoto e geoprocessamento. In: SIMPÓSIO BRASILEIRO DE SENSORIAMENTO REMOTO, 7, 1993, São José dos Campos. Anais... São José dos Campos: INPE, 1993. p. 73-80.

GÓES, C. A., MELLO FILHO, W. L., CARVALHO, M. Avaliação do desempenho de diferentes classificadores (Isoseg, Bhattacharyya, Maxver e Maxver-ICM), utilizando imagens CCD/CBERS-1 e ETM+/Landsat-7 fusionadas. Revista Ambi-Água. v. 1, n. 2, p. 80-89, 2006. 
GONÇALVEZ, M. L. Uma arquitetura neural modular para classificação de imagens multiespectrais de sensoriamento remoto. 1997. 112 f. Dissertação (Mestrado em Engenharia Elétrica) - Faculdade de Engenharia Elétrica e de Computação, Universidade Estadual de Campinas, Campinas, 1997.

INSTITUTO BRASILEIRO DE GEOGRAFIA E ESTATÍSTICA. Comentários. Disponível em: $<$ http://www.ibge.gov.br/home/estatistica/economia/pam/2007/comentario.pdf >. Acesso em: 18 dez. 2008.

INSTITUTO DE PESQUISAS TECNOLÓGICAS DO ESTADO DE SÃO PAULO. Mapa Geológico do Estado de São Paulo, São Paulo, Escala: 1000000. 1981.

INSTITUTO NACIONAL DE PESQUISAS ESPACIAIS. Divisão de Geração de Imagens (DGI-INPE). Catálogo de imagens. Disponível em: <http://www.dgi.inpe.br/>. Acesso em: 15 nov. 2008.

LILLESAND, T.; KIEFER, R. W. Remote sensing and image interpretation. 3rd ed. New York: John Wiley \& Sons, 1994.724 p.

MOREIRA, M. A. Fundamentos do sensoriamento remoto e metodologias de aplicação. São José dos Campos: Instituto Nacional de Pesquisas Espaciais, 2001. 250 p.

NOGUCHI, M. T. G. Um sistema baseado em regras fuzzy para classificação supervisionada de imagens multiespectrais de alta resolução. 2004. 150 f. Dissertação (Mestrado em Informática) - Universidade Federal do Paraná, Curitiba, 2004.

ORTIZ, J. L., FREITAS, M. I. C. Mapeamento do uso da terra, vegetação e impactos ambientais por meio de sensoriamento remoto e geoprocessamento. Geociências, v. 24, n. 1, p. 91-104, 2005.

SANCHES, I. D., GÜRTLER, S., FORMAGGIO, A. R. Discriminação de variedades de citros em imagens CCD/CBERS-2. Ciência Rural, v.38, n.1, p.103-108, 2008. 